\title{
Age differences in the neural representation of working memory revealed by multi-voxel pattern analysis
}

\author{
Joshua Carp ${ }^{1}$, Leon GmeindI ${ }^{2}$ and Patricia A. Reuter-Lorenz ${ }^{1}$ \\ Department of Psychology, University of Michigan, Ann Arbor, MI, USA \\ 2 Department of Psychological and Brain Sciences, Johns Hopkins University, Baltimore, MD, USA
}

\section{Edited by:}

Hauke R. Heekeren, Max Planck Institute for Human Development, Germany

\section{Reviewed by:}

Christian Fiebach, Goethe University Frankfurt, Germany

Leighton B. Hinkley, University of

California, USA

*Correspondence:

Joshua Carp, Department of Psychology, University of Michigan, 530 Church Street, Ann Arbor, MI 48109, USA.

e-mail:jmcarp@umich.edu
Working memory function declines across the lifespan. Computational models of aging attribute such memory impairments to reduced distinctiveness between neural representations of different mental states in old age, a phenomenon termed dedifferentiation. These models predict that neural distinctiveness should be reduced uniformly across experimental conditions in older adults. In contrast, the Compensation-Related Utilization of Neural Circuits Hypothesis ( $\mathrm{CRUNCH}$ ) model predicts that the distinctiveness of neural representations should be increased in older adults (relative to young adults) at low levels of task demand but reduced at high levels of demand. The present study used multi-voxel pattern analysis to measure the effects of age and task demands on the distinctiveness of the neural representations of verbal and visuospatial working memory. Neural distinctiveness was estimated separately for memory encoding, maintenance, and retrieval, and for low, medium, and high memory loads. Results from sensory cortex during encoding and retrieval were consistent with the dedifferentiation hypothesis: distinctiveness of visual cortical representations during these phases was uniformly reduced in older adults, irrespective of memory load. However, maintenance-related responses in prefrontal and parietal regions yielded a strikingly different pattern of results. At low loads, older adults showed higher distinctiveness than younger adults; at high loads, this pattern reversed, such that distinctiveness was higher in young adults. This interaction between age group and memory load is at odds with the dedifferentiation hypothesis but consistent with $\mathrm{CRUNCH}$. In sum, our results provide partial support for both dedifferentiation- and compensation-based models; we argue that comprehensive theories of cognitive aging must incorporate aspects of both models to fully explain complex patterns of age-related neuro-cognitive change.

Keywords: aging, working memory, dedifferentiation, compensation, multi-voxel pattern analysis, fMRI

\section{INTRODUCTION}

Computational models of cognitive aging posit that neural representations of different mental states become less distinctive in old age (Li et al., 2001), a view referred to as the dedifferentiation hypothesis. Consistent with this notion, behavioral studies show increases in correlations among cognitive and perceptual abilities across the adult lifespan (Lindenberger and Baltes, 1994; Baltes and Lindenberger, 1997). Furthermore, neuroimaging studies show that tasks associated with unilateral brain activation in young adults evoke bilateral activation in older adults (Reuter-Lorenz et al., 2000; Cabeza et al., 2002; Duverne et al., 2009). Similarly, neural specialization in object-sensitive visual cortex decreases in old age (Park et al., 2004). These findings imply that different mental operations increasingly rely on shared neural substrates in the aging brain.

However, age differences in the distinctiveness of neural representations may not be uniform across experimental conditions. In particular, the Compensation-Related Utilization of Neural Circuits Hypothesis (CRUNCH) model predicts that age differences in neural engagement should vary with the level of task demand (ReuterLorenz and Cappell, 2008). According to CRUNCH, declining neural efficiency leads older adults to recruit more neural resources than young adults at low levels of task demand. However, as task demands increase, older adults reach a resource ceiling, resulting in under-activation relative to young adults. Results from several studies of working memory conform to this pattern (Mattay et al., 2006; Schneider-Garces et al., 2010), including a previously published analysis of a subset of the data described here (Cappell et al., 2010). However, the analyses used by these studies did not permit measurement of the distinctiveness between neural representations, focusing instead on age differences in overall activation.

How, according to CRUNCH, should neural distinctiveness change with age and task demands? As task demands increase, subjects increasingly rely on specialized neural resources (Smith et al., 1996; Jonides et al., 1997). However, when task demands exceed the capacity of such specialized mechanisms, additional task-general resources may be recruited (Reuter-Lorenz et al., 1999; Rypma et al., 1999). Thus, neural representations of distinct tasks should be highly discriminable when task demands approach the capacity of specialized neural resources: under such conditions, each task should strongly recruit a set of domain-specific mechanisms. In contrast, when task demands are lower than the capacity of such specialized resources, representations of the two tasks should be less discriminable, as neither set of specialized mechanisms is strongly recruited under these conditions. Similarly, task representations should be less distinctive when demands exhaust 
the capacity of task-specific resources: under such conditions, both tasks should recruit overlapping sets of domain-general neural resources.

Because older adults are thought to reach their resource limits at lower levels of task demand than young adults (Cappell et al., 2010; Schneider-Garces et al., 2010), CRUNCH predicts that the distinctiveness of neural representations should be greater in older adults than young adults when task demands are low. In contrast, when task demands are high, CRUNCH predicts that neural distinctiveness should be higher in young adults than in older adults. While the dedifferentiation hypothesis and CRUNCH predict different patterns of age-related change in neural distinctiveness, the two models are not mutually exclusive. For example, some mental operations (and their neural underpinnings) may be explained best by agerelated dedifferentiation; others may follow the pattern predicted by CRUNCH. Indeed, previous research has offered the intriguing possibility that age-related dedifferentiation in sensory cortex degrades inputs to higher-order processes, leading to compensation in prefrontal and parietal regions (Park and Reuter-Lorenz, 2009).

Although many studies have investigated the effects of aging on neural recruitment, nearly all of these studies relied on univariate measures of brain activation. However, the relationship between such univariate tests and the distinctiveness of neural representations remains unclear. In particular, neural representations of different mental states may be highly distinctive even when these states evoke indistinguishable univariate activation (Peelen et al., 2006; Dinstein et al., 2008). In contrast, recently developed techniques focusing on multi-voxel activation patterns permit more direct investigations of representational distinctiveness (Haynes and Rees, 2006; Norman et al., 2006). Consistent with the dedifferentiation hypothesis, recent studies using this multi-voxel pattern analysis (MVPA) of AMRI data show that neural representations of visual object categories (faces, houses, pseudo-words, and chairs) become less distinctive in old age (Carp et al., 2010; Park et al., 2010). However, in contrast to the present study, these reports focused on visual perception and provide little insight into age differences in high-level cognition. Further, these studies did not systematically vary levels of task demand, precluding tests of the CRUNCH model.

To compare the predictions of the dedifferentiation hypothesis and CRUNCH, the present study used MVPA to assess the effects of age and task demands on the distinctiveness of the neural representations of verbal and visuospatial working memory. Healthy young and older adults performed verbal and visuospatial working memory tasks in separate scanning runs. Univariate analysis of the verbal working memory data is described in a separate report (Cappell et al., 2010). Here, distinctiveness between the two memory tasks was evaluated separately during memory encoding, maintenance, and retrieval for low, medium, and high memory loads. Following $\mathrm{Li}$ and Sikström (2002), we define the neural representation of a mental state as the pattern of activation elicited by that state; neural representations of different states are said to be distinctive to the extent that one can be distinguished from the other.

\section{MATERIALS AND METHODS PARTICIPANTS}

Eighteen young adults (mean age 20.9 years, SD 1.63 years, range $18-25,10$ female) and 23 older adults (mean age 68.3 years, SD 6.67, range 61-82, 13 female) participated in the experiment.
All participants were right-handed, with normal or correctedto-normal vision. Participants had no history of head trauma or neurological or psychiatric illness, and a minimum MiniMental State Exam (MMSE) score of 25; older adults had a mean MMSE score of 29.2. Informed consent was obtained from all participants; all procedures were approved by the University of Michigan's Institutional Review Board.

\section{EXPERIMENTAL DESIGN}

Participants performed delayed verbal and visuospatial itemrecognition working memory (WM) tasks in separate runs while fMRI data were acquired. Both tasks were adapted from ReuterLorenz et al. (2000); the verbal WM task is also described in a previous report on these data (Cappell et al., 2010). Each trial comprised three phases: encoding, maintenance, and retrieval. To minimize colinearity between task phases, durations of the maintenance phase and the inter-trial interval were jittered across trials (Dale, 1999).

During the encoding phase (1.5 s), participants were presented with four, five, or seven uppercase letters (verbal task) or the spatial locations of one, two, or three filled circles (visuospatial task). Letters were evenly spaced along an imaginary circle with a radius of $5^{\circ}$ centered on the fixation point; spatial locations of the target letters were irrelevant, and there was no requirement for subjects to remember the locations of letters. Circles appeared at randomly chosen positions along imaginary circles with radii of $2.5^{\circ}, 5^{\circ}$, or $7.5^{\circ}$. The maintenance phase was an unfilled delay with a variable duration of $4 \mathrm{~s}(25 \%), 6 \mathrm{~s}(25 \%), 8 \mathrm{~s}(25 \%)$, or $10 \mathrm{~s}(25 \%)$. Finally, during the probe phase (1.5 s), a single lowercase letter (verbal task) or circle (visuospatial task) was presented, and participants indicated whether the probe stimulus belonged to the current memory set (match trials; 50\%) or did not (non-match trials; 50\%). In the verbal task, probe letters always appeared at fixation. Each trial was followed by a variable fixation interval of 1.5 s (50\%), 3 s (25\%), 4.5 s (12.5\%), or 6 s (12.5\%). Participants were instructed to respond as accurately as possible and to fixate a centrally presented red dot throughout each run.

Participants completed four runs of the verbal task and four runs of the visuospatial task. Runs were presented in ABBABAAB order; the tasks designated by $\mathrm{A}$ and $\mathrm{B}$ were counterbalanced across subjects. Each run comprised 24 trials presented in random order; thus, each participant completed 96 trials for each of the two tasks. Fixation intervals of $20 \mathrm{~s}$ duration were presented at the beginning of each run, and after the 8th and 16th trials. All experimental stimuli were presented using EPrime software (Psychology Software Inc., Pittsburgh, PA, USA).

\section{fMRI DATA ACQUISITION}

Images were acquired using a 3T whole-body MRI scanner (General Electric). Blood oxygenation level dependent (BOLD) images were acquired using a spiral sequence in 43 contiguous axial 3 -mm slices, with an in-plane resolution of $3.44 \mathrm{~mm} \times 3.44 \mathrm{~mm}$ $\left(\mathrm{TR}=2 \mathrm{~s}, \mathrm{TE}=30 \mathrm{~ms}\right.$, flip angle $=90^{\circ}, \mathrm{FOV}=22 \mathrm{~cm}$, in-plane matrix $=64 \times 64$ voxels). High-resolution T1-weighted images with the same orientation as the functional scans were collected at the end of the session $\left(\mathrm{TR}=10 \mathrm{~ms}, \mathrm{TE}=3.4 \mathrm{~ms}\right.$, flip angle $=23^{\circ}$, $\mathrm{FOV}=24 \mathrm{~cm}$, matrix $=256 \times 256$ voxels $)$. 


\section{DATA ANALYSIS}

Preprocessing and model estimation were conducted using SPM5 software (Wellcome Department of Cognitive Neurology, London, UK, www.fil.ion.ucl.ac.uk). Subsequent analysis was performed using custom routines implemented in MATLAB (MathWorks, Inc., Natick, MA, USA) and the R statistical computing language (R Foundation for Statistical Computing, Vienna, Austria).

\section{fMRI preprocessing}

Functional data were corrected for differences in slice time acquisition and realigned to the first volume using standard functions in SPM5. No spatial normalization or smoothing was applied prior to multivariate analysis (Haxby et al., 2001).

\section{Multi-voxel pattern analysis}

We used MVPA to measure age differences in the distinctiveness of neural representations of verbal and visuospatial working memory. Neural distinctiveness was estimated using a correlation distance metric (Haxby et al., 2001; Haushofer et al., 2008; Carp et al., 2010). We selected this metric over alternative multivariate techniques (e.g., support vector machines, neural network classifiers) because its logic and implementation are relatively simple and because it does not require the optimization of as many free parameters. We also note that previous research has documented similar effects using different multivariate analyses. For example, Park et al. (2010) showed that correlations between neural distinctiveness and behavioral performance were highly similar whether distinctiveness was measured using correlation distance (as in the present study) or using support vector machines.

We first estimated the neural response for each working memory condition (verbal and visuospatial), task phase (encoding, maintenance, and retrieval), and memory load (low, medium, and high). Activation for even- and odd-numbered runs was estimated using separate regressors (Haxby et al., 2001). Neural responses related to encoding, maintenance, and retrieval were modeled using separate event-related regressors (Postle et al., 2000); this analysis was carried out using the General Linear Model (Friston et al., 1995) as implemented in SPM5. Only correct trials were included in the analysis; incorrect trials were modeled separately as a nuisance covariate.

Next, we used the activation estimates derived from the GLM analysis described above to assess the distinctiveness between distributed representations of the verbal and visuospatial working memory tasks. To do so, we compared correlations across voxels within and between the verbal and visuospatial tasks, across evenand odd-numbered runs. The distinctiveness between verbal and visuospatial tasks for any given set of voxels was defined as the difference between the mean Fisher-transformed correlations across those voxels' $\beta$-values within and between the two tasks (Haxby et al., 2001; Haushofer et al., 2008):

$$
\begin{aligned}
& \operatorname{corr}_{\text {within-task }}=\left(\operatorname{corr}_{\text {verbaleven, verbalodd }}+\operatorname{corr}_{\text {spatialeven, spatialodd }}\right) / 2 \\
& \operatorname{corr}_{\text {between-task }}=\left(\operatorname{corr}_{\text {verbaleven, spatialodd }}+\operatorname{corr}_{\text {spatialeven, verbalodd }}\right) / 2 \\
& \text { distinctiveness }=\operatorname{corr}_{\text {within-task }}-\text { corr }_{\text {between-task }}
\end{aligned}
$$

To minimize the contribution of potential age differences in BOLD variability to our results, we used $\beta$-values, which are not scaled by model error, rather than $t$-values, for this analysis (Rypma and D'Esposito, 2000). Positive distinctiveness scores indicate that activation patterns distinguished between memory conditions; distinctiveness scores of zero indicate that activation patterns were uninformative with regard to memory conditions.

To generate whole-brain maps of pattern distinctiveness, we combined the correlation analysis described above with a multivariate searchlight procedure (Kriegeskorte et al., 2006). For each voxel in the brain, we identified all voxels within a 12 -mm-radius sphere centered on that voxel. This radius was selected to maximize neural distinctiveness across all conditions and age groups (and, thus, to maximize sensitivity to detect between-condition differences in distinctiveness). Next, we calculated the distinctiveness between verbal and visuospatial memory conditions across this group of voxels. The resulting neural distinctiveness score was then entered as the value for the center voxel. This procedure was iterated across all voxels in the brain, yielding a whole-brain map of neural distinctiveness between the two memory tasks. The neural distinctiveness value at each voxel reflects the discriminability between tasks for the local pattern of activation centered on that voxel. Separate searchlight maps were estimated for each trial phase and memory load. These maps were subsequently normalized into MNI space and averaged within age groups.

\section{Random-effects analysis}

For each of the three trial phases (encoding, maintenance, and retrieval), voxel-wise neural distinctiveness maps were submitted to a two-way mixed ANOVA including a between-subjects factor of age group (young, old) and a within-subjects factor of memory load (low, medium, and high). Voxel-wise $F$-maps were thresholded at a height threshold of $p<0.005$ and an extent threshold of 50 contiguous voxels (e.g., Daselaar et al., 2003; Persson et al., 2007; Miller et al., 2008).

\section{RESULTS}

\section{BEHAVIORAL DATA}

Participants' reaction time (RT) and accuracy data were analyzed using separate mixed ANOVAs with within-subjects factors of task (verbal, visuospatial) and load (low, medium, and high) and a between-subjects factor of age group (young, old). Incorrect and omitted responses were excluded from the RT analysis. RT and accuracy data are presented in Figures $\mathbf{1}$ and 2 , respectively.

Reaction time data revealed a significant main effect of load: RT increased with increasing memory load $[F(2,39)=86.85$, $p<0.001]$. The main effect of age group was also significant: older adults responded more slowly than younger adults $[F(1$, $39)=22.75, p<0.001]$. We also observed a significant main effect of memory task, such that responses were slower for the verbal task than for the visuospatial task $[F(1,39)=22.21, p<0.001]$. Finally, we found a significant interaction between age group and memory load: the effect of age on RT increased with memory load $[F(2,78)=5.62, p=0.0052]$. No additional RT effects reached significance.

Accuracy data showed a significant main effect of memory load, such that accuracy decreased with increasing load $[F(2,39)=67.88, p<0.001]$. We also observed a significant interaction between age group and task $[F(2,39)=4.34$, $p=0.016]$ : older adults showed lower accuracy than young adults 


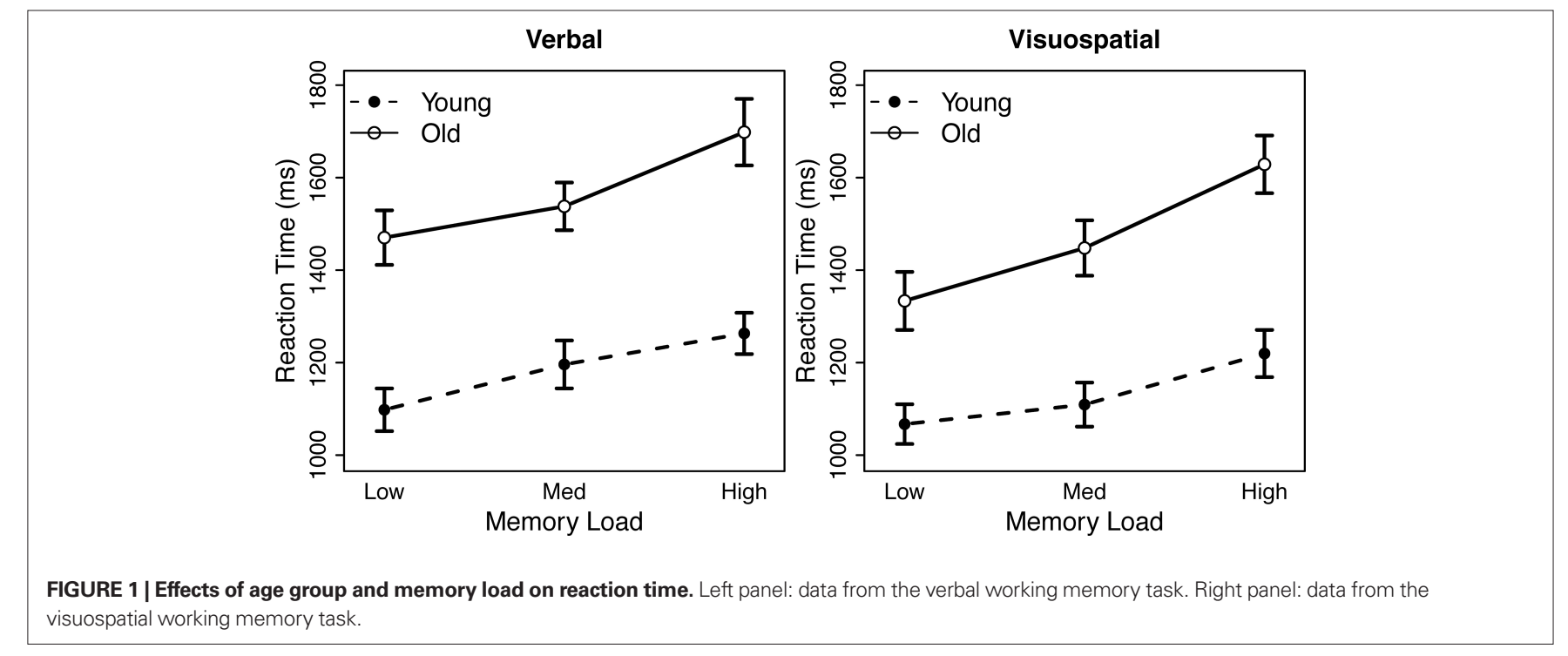

FIGURE 2 | Effects of age group and memory load on response accuracy. Left panel: data from the verbal working memory task. Right panel: data from the
visuospatial working memory task.

for the verbal task $[F(1,39)=3.96, p=0.054]$, but not for the visuospatial task $[F<1, n s]$. No additional accuracy effects were significant.

\section{fMRI DATA}

\section{Encoding phase}

According to the dedifferentiation hypothesis, the distinctiveness of neural representations should be uniformly reduced in old age. To test this view, we measured overall age differences in distinctiveness during memory encoding. Voxel-wise analysis revealed significant main effects of age group in early visual areas, including left striate cortex, right lingual gyrus, and bilateral inferior occipital gyrus (Table 1; Figure 3). We also observed significant effects of age in regions that are thought to play important roles in working memory performance, including left inferior frontal gyrus, right middle frontal gyrus, and left inferior parietal lobule. Inspection of these clusters revealed reduced neural distinctiveness in older adults for each of these clusters (Figure 3). Critically, no regions showed higher neural distinctiveness in older adults than in young adults.

We observed significant interactions between age group and memory load in right middle frontal gyrus, left middle temporal gyrus, and anterior cingulate cortex (Table 1; Figure 4). Inspection of these clusters revealed a consistent pattern, such that neural distinctiveness increased with memory load in younger adults (all cluster simple effects, $p s \leq 0.07$ ) but decreased with load in older adults (all cluster simple effects, $p s \leq 0.05$; Figure 4). Critically, distinctiveness in these regions was equivalent across age groups at low memory load but significantly reduced in older adults at high memory load (all cluster simple effects, $p s \leq 0.05$ ). 
Table 1 | Encoding-related neural distinctiveness.

\begin{tabular}{|c|c|c|c|c|c|c|c|c|c|}
\hline \multirow[t]{3}{*}{ Region } & \multirow{3}{*}{$\begin{array}{l}\text { Number } \\
\text { of voxels }\end{array}$} & \multicolumn{3}{|c|}{ MNI coordinates } & \multicolumn{4}{|c|}{ Neural distinctiveness } & \multirow[t]{3}{*}{ Statistics } \\
\hline & & \multirow[b]{2}{*}{$X$} & \multirow[b]{2}{*}{$Y$} & \multirow[b]{2}{*}{$Z$} & \multirow[b]{2}{*}{ Age group } & \multicolumn{3}{|c|}{ Memory load } & \\
\hline & & & & & & Low & Med & High & \\
\hline \multicolumn{10}{|l|}{ MAIN EFFECT OF AGE } \\
\hline \multirow[t]{2}{*}{ L. inferior frontal gyrus } & 431 & -47 & 28 & 15 & Young & 0.16 & 0.19 & 0.28 & $F(1,117)=23.46, p<0.001$ \\
\hline & & & & & Old & 0.04 & 0.05 & 0.03 & \\
\hline \multirow[t]{2}{*}{ R. middle frontal gyrus } & 56 & 24 & 48 & 12 & Young & 0.07 & 0.11 & 0.11 & $F(1,117)=13.75, p<0.001$ \\
\hline & & & & & Old & 0.02 & 0.01 & -0.05 & \\
\hline \multirow[t]{2}{*}{ L. inferior parietal lobule } & 204 & -55 & -31 & 39 & Young & 0.07 & 0.13 & 0.14 & $F(1,117)=10.78, p<0.001$ \\
\hline & & & & & Old & 0.03 & 0.01 & -0.01 & \\
\hline \multirow[t]{2}{*}{ L. striate cortex } & 1144 & -7 & -100 & -3 & Young & 0.16 & 0.31 & 0.19 & $F(1,117)=25.65, p<0.001$ \\
\hline & & & & & Old & 0.02 & 0.09 & 0.06 & \\
\hline \multirow[t]{2}{*}{ L. inferior occipital gyrus } & 1144 & -31 & -89 & -18 & Young & 0.13 & 0.20 & 0.16 & $F(1,117)=18.36, p<0.001$ \\
\hline & & & & & Old & 0.04 & 0.05 & 0.05 & \\
\hline \multirow[t]{2}{*}{ R. lingual gyrus } & 1144 & 17 & -86 & -12 & Young & 0.25 & 0.21 & 0.27 & $F(1,117)=18.98, p<0.001$ \\
\hline & & & & & Old & 0.13 & 0.09 & 0.08 & \\
\hline \multirow[t]{2}{*}{ R. inferior occipital gyrus } & 1144 & 38 & -86 & -15 & Young & 0.19 & 0.16 & 0.15 & $F(1,117)=19.94, p<0.001$ \\
\hline & & & & & Old & 0.07 & 0.04 & 0.00 & \\
\hline \multicolumn{10}{|c|}{ AGE BY LOAD INTERACTION } \\
\hline \multirow[t]{2}{*}{ R. middle frontal gyrus } & 267 & 41 & 34 & 18 & Young & 0.07 & 0.04 & 0.19 & $F(2,117)=10.52, p<.001$ \\
\hline & & & & & Old & 0.08 & 0.07 & -0.01 & \\
\hline \multirow[t]{2}{*}{ L. middle temporal gyrus } & 103 & -41 & -65 & 30 & Young & 0.04 & 0.20 & 0.20 & $F(2,117)=9.11, p<0.001$ \\
\hline & & & & & Old & 0.10 & 0.05 & 0.01 & \\
\hline \multirow[t]{2}{*}{ Anterior cingulate cortex } & 66 & 10 & 31 & 27 & Young & 0.06 & 0.01 & 0.18 & $F(2,117)=7.17, p<0.001$ \\
\hline & & & & & Old & 0.03 & 0.08 & 0.01 & \\
\hline
\end{tabular}

\section{Maintenance phase}

Next, we measured the effects of age group and memory load on neural distinctiveness during the maintenance phase. In contrast to the encoding phase, overall neural distinctiveness did not vary with age group: no regions showed a significant main effect of age group.

However, we observed age group by memory load interactions across several prefrontal and parietal regions, including orbitofrontal cortex and bilateral superior and inferior frontal gyrus (Table 2; Figure 5). The left inferior frontal gyrus cluster showed partial overlap with the main effect of age observed during memory encoding (Table 1; Figure 3). Inspection of these results showed a consistent pattern across regions. In each cluster, neural distinctiveness increased with memory load in young adults (all cluster $p s \leq 0.01$ ). In older adults, however, neural distinctiveness tended to decrease with increasing memory load (orbitofrontal cortex, left superior frontal gyrus, left inferior frontal gyrus, right inferior frontal gyrus: $p s \leq 0.05$; right superior frontal gyrus, left precuneus: $n s$; Figure 5). Thus, older adults showed greater neural distinctiveness than young adults at low loads (all cluster $p s \leq 0.05$ ) and less distinctiveness than young adults at high loads (all cluster $p s \leq 0.05)$. These interactions mirror effects observed in the behavioral data: older adults showed the greatest RT impairment at high memory loads.

\section{Retrieval phase}

Finally, we examined retrieval-phase distinctiveness between verbal and visuospatial conditions as a function of age group and memory load. We observed a significant main effect of age group in left extrastriate cortex, such that neural distinctiveness was reduced in older adults (Table 3). This cluster showed substantial overlap with the main effect of age observed during memory encoding (Table 1; Figure 3). No age by load interactions reached significance in this analysis.

\section{DISCUSSION}

The present study measured age differences in the neural representations of memory encoding, maintenance, and retrieval using MVPA. Results from sensory cortex during memory encoding and retrieval were consistent with age-related neural dedifferentiation: older adults showed reduced distinctiveness between verbal and visuospatial memory conditions, regardless of memory load (Table 1; Figure 3). In contrast, results from memory maintenance were difficult to reconcile with the dedifferentiation hypothesis but consistent with the CRUNCH model (ReuterLorenz and Cappell, 2008). During the maintenance phase, neural distinctiveness in prefrontal and parietal regions increased with memory load in young adults. In older adults, this pattern was absent or even reversed. Thus, relative to young adults, older 


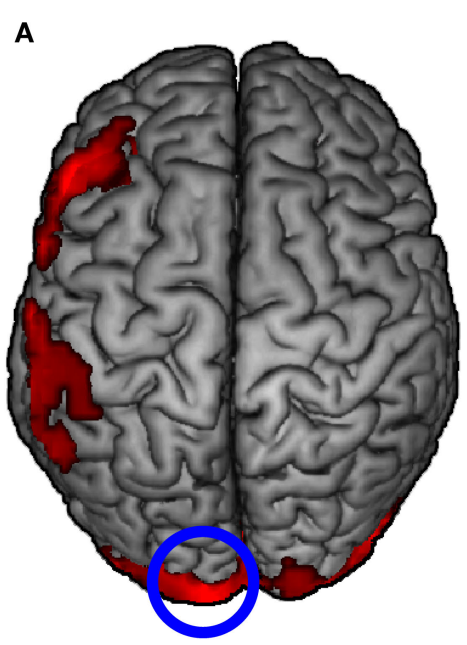

C

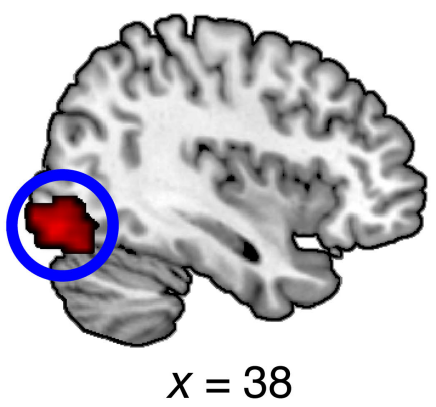

FIGURE 3 | Main effect of age group during working memory encoding. See also Table 1, Main effect of age. (A) Older adults showed decreased distinctiveness between verbal and visuospatial WM tasks in prefrontal, parietal, and sensory cortex. Left striate cortex is highlighted. (B) Neural
B

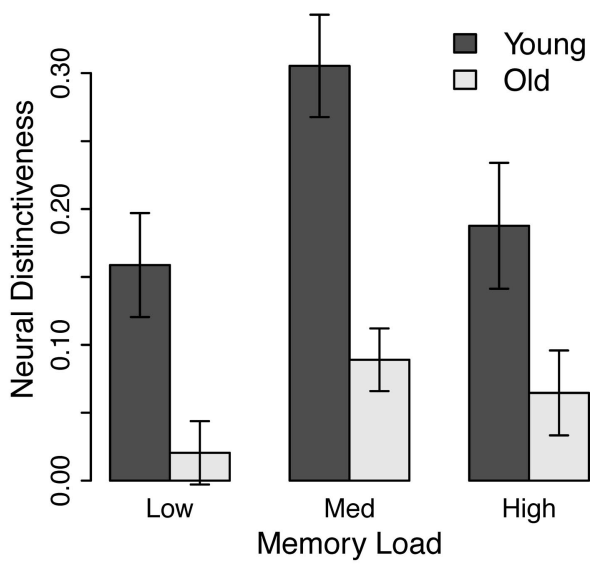

D

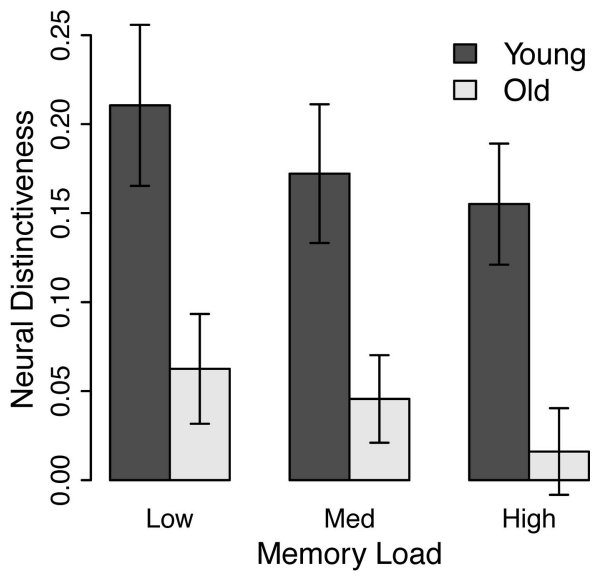

distinctiveness scores from left striate cortex. (C) Older adults also showed decreased neural distinctiveness in right inferior occipital gyrus ( $x=38$ ). (D) Neural distinctiveness scores from right inferior occipital gyrus. adults showed increased maintenance-related distinctiveness at low memory loads but decreased distinctiveness at high memory loads (Table 2; Figure 5).

Results from visual cortex are broadly consistent with previous research on age-related dedifferentiation. Previous studies have documented age differences in sensory regions during working memory tasks (for reviews, see Reuter-Lorenz and Lustig, 2005; Park and Reuter-Lorenz, 2009). The present results corroborate and extend these reports, suggesting that age differences in sensory activity reflect, at least in part, changes in representational distinctiveness. Our findings dovetail with recent studies showing age-related declines in the distinctiveness of neural representations of visual objects (Carp et al., 2010; Park et al., 2010). Our results are also consistent with single-unit recording studies showing inefficient perceptual representations of simple visual stimuli in senescent monkeys (Schmolesky et al., 2000; Leventhal et al., 2003) and cats (Hua et al., 2006). Furthermore, these findings confirm prior research showing that object representations in prefrontal and parietal cortex become less discriminable in old age
(Carp et al., 2010), suggesting that age-related dedifferentiation is not restricted to ventral visual cortex. Finally, our results are generally consistent with reports of hemispheric specialization of motor (Hutchinson et al., 2002; Mattay et al., 2002) and auditory (Bellis et al., 2000) representations in old age (although, to our knowledge, no published work has investigated age differences in the distinctiveness of these representations using the multivariate approach described here).

However, results from memory maintenance do not support the view that representational distinctiveness is uniformly reduced in older adults. Indeed, no brain regions exhibited a significant main effect of age group during the maintenance phase. Instead, relative to young adults, older adults showed increased distinctiveness at low memory loads and decreased distinctiveness at high loads (Figure 5). Consistent with this observation, analysis of the reaction time data revealed that older adults were most impaired at high memory loads. These results are consistent with CRUNCH, which posits that older adults must recruit more neural resources than young adults to maintain performance for a given level of task dif- 


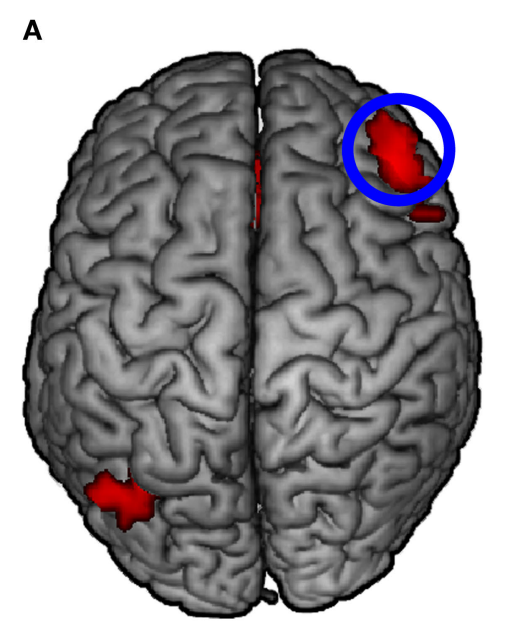

FIGURE 4 | Age group by load interaction during working memory encoding. See also Table 1, Age by load interaction. (A) Neural distinctiveness increased with load in younger adults but decreased with load in older adults in
B

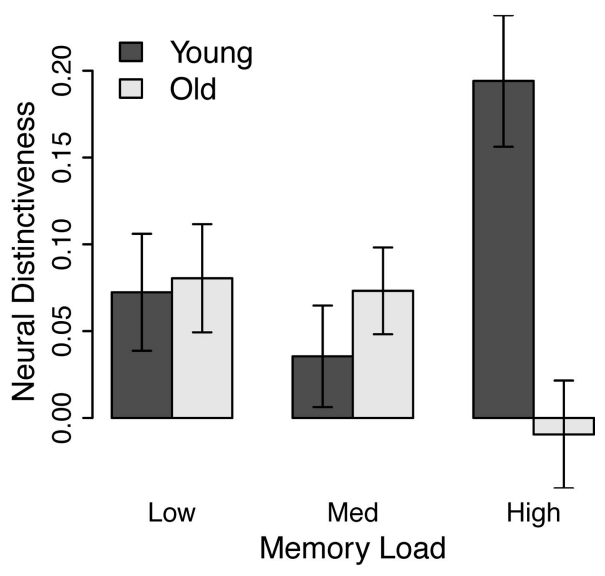

right middle frontal gyrus, anterior cingulate cortex, and left middle temporal gyrus. Middle frontal gyrus is highlighted. (B) Neural distinctiveness scores from right middle frontal gyrus.

Table 2 | Maintenance-related neural distinctiveness.

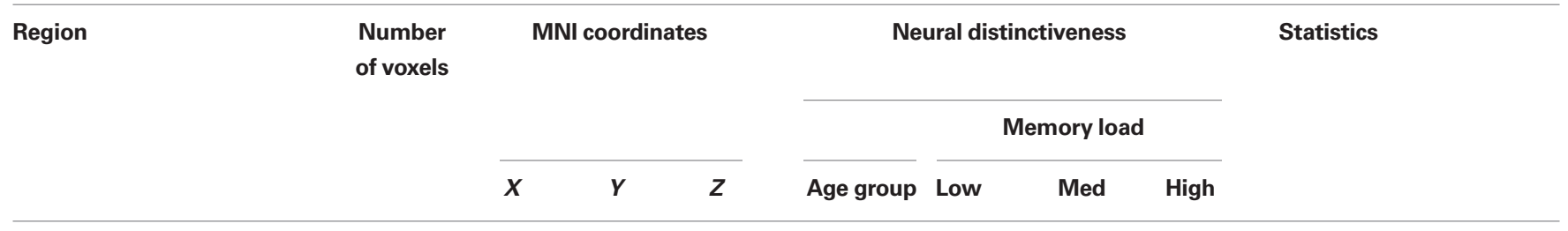

\section{MAIN EFFECT OF AGE}

No significant clusters

\begin{tabular}{|c|c|c|c|c|c|c|c|c|c|}
\hline \multicolumn{10}{|c|}{ AGE BY LOAD INTERACTION } \\
\hline \multirow[t]{2}{*}{ Orbitofrontal cortex } & 523 & 0 & 55 & -15 & Young & -0.02 & 0.04 & 0.18 & $F(2,117)=13.48, p<0.001$ \\
\hline & & & & & Old & 0.05 & 0.05 & 0.00 & \\
\hline \multirow[t]{2}{*}{ L. superior frontal gyrus } & 171 & -38 & 17 & 54 & Young & -0.03 & 0.09 & 0.17 & $F(2,117)=11.72, p<0.001$ \\
\hline & & & & & Old & 0.12 & 0.03 & 0.03 & \\
\hline \multirow[t]{2}{*}{ L. inferior frontal gyrus } & 323 & -47 & 10 & 21 & Young & -0.02 & 0.07 & 0.11 & $F(2,117)=9.48, p<0.001$ \\
\hline & & & & & Old & 0.09 & 0.03 & 0.03 & \\
\hline \multirow[t]{2}{*}{ L. inferior frontal gyrus } & 161 & -24 & 31 & -3 & Young & -0.02 & 0.14 & 0.21 & $F(2,117)=7.87, p<0.001$ \\
\hline & & & & & Old & 0.13 & 0.06 & 0.07 & \\
\hline \multirow[t]{2}{*}{ R. superior frontal gyrus } & 429 & 41 & 38 & 33 & Young & 0.05 & 0.06 & 0.21 & $F(2,117)=10.24, p<0.001$ \\
\hline & & & & & Old & 0.13 & 0.11 & 0.09 & \\
\hline \multirow[t]{2}{*}{ R. inferior frontal gyrus } & 429 & 38 & 24 & 12 & Young & -0.06 & 0.03 & 0.14 & $F(2,117)=15.46, p<.001$ \\
\hline & & & & & Old & 0.09 & 0.00 & 0.01 & \\
\hline \multirow[t]{2}{*}{ L. precuneus } & 85 & -17 & -58 & 33 & Young & -0.02 & 0.07 & 0.16 & $F(2,117)=8.45, p<0.001$ \\
\hline & & & & & Old & 0.08 & 0.06 & 0.05 & \\
\hline
\end{tabular}

ficulty. Thus, when task demands are low, older adults engage more task-specific resources than young adults. However, older adults are more likely than young adults to reach their resource limitations when task demands are high, leading to increased reliance on auxiliary task-general mechanisms and, in consequence, decreased distinctiveness between task representations. These results show that dedifferentiation is not a general property of the aging brain: depending on the level of task demand, older adults can show higher or lower neural differentiation than young adults.

These results complement and extend previous studies of age by memory load interactions on neural recruitment. For example, studies of working memory using univariate analysis of fMRI 

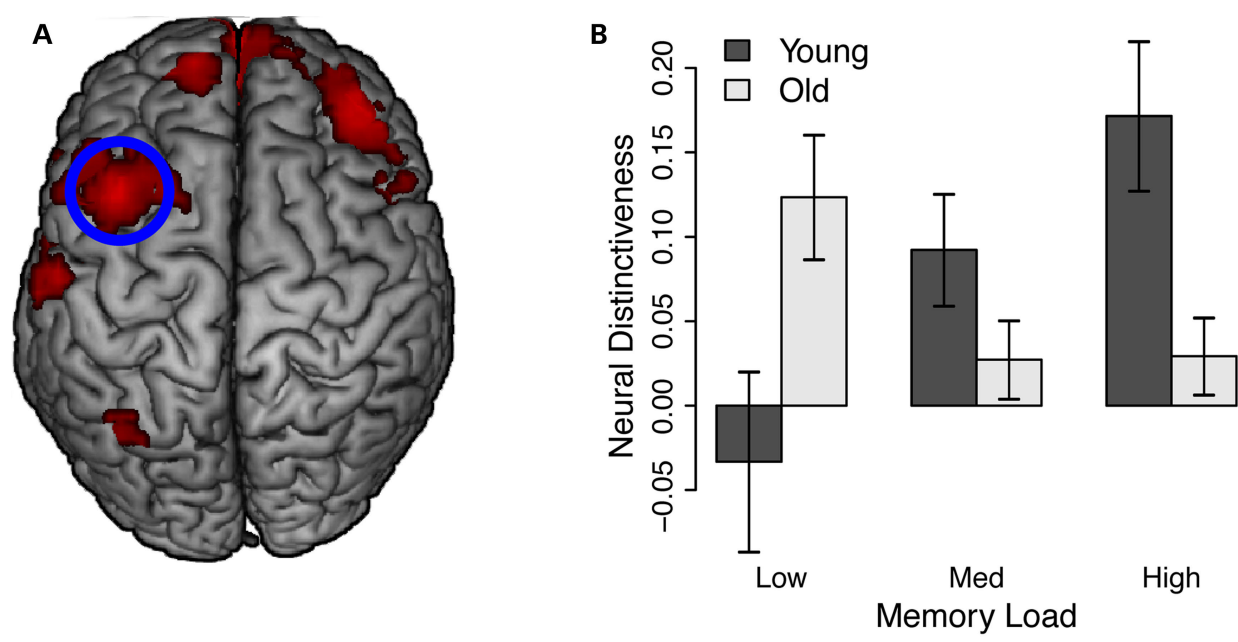

C

D

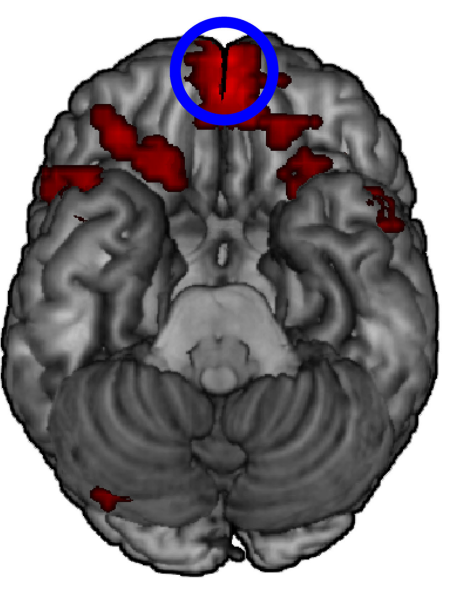

FIGURE 5 |Age group by load interaction during working memory maintenance. See also Table 2, Age by load interaction. (A) Neural distinctiveness increased with load in younger adults but decreased with load in older adults across several prefrontal and parietal clusters. Left superior frontal

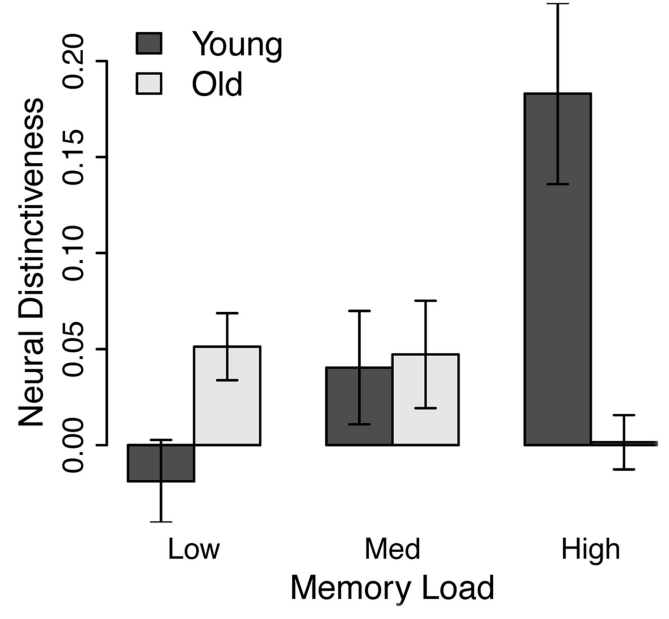

gyrus is highlighted. (B) Neural distinctiveness scores from left superior frontal gyrus. Further descriptions of these results are given in Table 2. (C) Age by load interactions along the ventral surface of the brain. Orbitofrontal cortex is highlighted. (D) Neural distinctiveness scores from orbitofrontal cortex.

Table 3 | Retrieval-related neural distinctiveness.

\begin{tabular}{|c|c|c|c|c|c|c|c|c|c|}
\hline \multirow[t]{3}{*}{ Region } & \multirow{3}{*}{$\begin{array}{l}\text { Number } \\
\text { of voxels }\end{array}$} & \multicolumn{3}{|c|}{ MNI coordinates } & \multicolumn{4}{|c|}{ Neural distinctiveness } & \multirow[t]{3}{*}{ Statistics } \\
\hline & & \multirow[b]{2}{*}{$x$} & \multirow[b]{2}{*}{$Y$} & \multirow[b]{2}{*}{$z$} & \multicolumn{4}{|c|}{ Memory load } & \\
\hline & & & & & Age group & Low & Med & High & \\
\hline \multicolumn{10}{|c|}{ MAIN EFFECT OF A AGE } \\
\hline L. extrastriate cortex & & & & & Old & 0.09 & 0.05 & 0.08 & \\
\hline
\end{tabular}

No significant clusters 
(Mattay et al., 2006; Cappell et al., 2010; Schneider-Garces et al., 2010) and EEG (McEvoy et al., 2001) data have documented agerelated over-activation at low memory load and under-activation at high memory load. Unlike the present findings, these previous results do not speak to the distinctiveness or fidelity of task representations. Nevertheless, consistent with our results, they show that increases in task demand can have opposing effects on neural recruitment in young and elderly populations.

As reviewed above, previous studies have reported neuroimaging evidence consistent with age-related dedifferentiation (Carp et al., 2010; Park et al., 2010) and with the CRUNCH model (Mattay et al., 2006; Cappell et al., 2010). However, prior support for the two models has been obtained in different studies, using different subjects and experimental paradigms. Here, in a single experiment, we show that sensory responses during memory encoding and retrieval were consistent with age-related dedifferentiation, whereas prefrontal and parietal responses during memory maintenance supported the CRUNCH model. Thus, we argue that healthy aging has divergent effects on different mental operations that subserve working memory. These results are consistent with a recent review by Rajah and D'Esposito (2005), which showed that different regions of prefrontal cortex undergo different patterns of age-related change. While both dedifferentiation and the CRUNCH model can account for certain aspects of our results, neither theory is sufficient to explain the overall pattern of results.

The present study investigated age differences in the distinctiveness of intra-regional representations, focusing on fine-scale activation patterns in local neighborhoods of voxels. In contrast, previous neuroimaging studies have documented age-related dedifferentiation of inter-regional neural representations, focusing on differences between distant brain regions (for a review, see Reuter-Lorenz and Park, 2010). In particular, as reviewed by Cabeza's (2002) hemispheric asymmetry reduction in older adults (HAROLD) model, many studies have reported that tasks that evoke lateralized activation in young adults tend to evoke bilateral activation in older adults. The age-related reductions in neural distinctiveness that we observed during memory encoding and retrieval accord with the HAROLD model: both show that the neural substrates of different cognitive states become more similar in old age. However, the present results may not reflect the same phenomenon documented by HAROLD: age-related dedifferentiation of intra- and inter-regional activation patterns may or may not stem from a common mechanism.

\section{REFERENCES}

Baltes, P. B., and Lindenberger, U. (1997). Emergence of a powerful connection between sensory and cognitive functions across the adult life span: a new window to the study of cognitive aging? Psychol. Aging 12, 12-21.

Bellis, T. J., Nicol, T., and Kraus, N. (2000). Aging affects hemispheric asymmetry in the neural representation of speech sounds. J. Neurosci. 20 , 791-797.

Cabeza, R.(2002).Hemispheric asymmetry reduction in older adults: the HAROLD model. Psychol. Aging 17, 85-100.

In contrast to prior reports, the present study focuses on age differences in neural representation, rather than differences in overall activation. The interpretation of age differences in activation has proven contentious: age-related over-activation in frontal and parietal cortex has been hypothesized to reflect both compensation and impairment (Reuter-Lorenz and Lustig, 2005). In contrast, MVPA measures the information present in patterns of neural activation (Haxby et al., 2001; Haynes and Rees, 2006; Norman et al., 2006), simplifying the interpretation of age differences. If neural distinctiveness is reduced in older adults, we can conclude that activation patterns in these subjects convey less information than those in young adults; if older adults show increased distinctiveness, we can conclude that their activation patterns are more informative than those of young adults. Thus, the use of MVPA in this study helps to mitigate the interpretive ambiguities associated with the analysis of age differences in average BOLD response. Nevertheless, MVPA has its limitations: while this method can reveal whether neural activation patterns are discriminable, it does not explain the way these activations differ, or the computational mechanisms underlying the observed results.

While our analysis focused on the effects of task demand in the context of working memory, our results may generalize to other processes as well. For example, increasing demand on task-switching or interference resolution mechanisms may also lead to decreased neural distinctiveness in older adults. Future studies should examine these issues to determine the generality of our results.

In summary, the present study charts the effects of healthy aging on neural representations of working memory. Our results provide partial support for both age-related dedifferentiation and the CRUNCH model. Critically, though, neither model can explain the full range of effects present in the data. We suggest that hybrid models, incorporating aspects of both dedifferentiation and compensation, will be necessary to account for the complex pattern of neuro-cognitive change associated with healthy aging.

\section{ACKNOWLEDGMENTS}

This research was supported by an NDSEG fellowship to Joshua Carp, an NSF Graduate Research Fellowship to Leon Gmeindl, and research grant R01-AG18286 to Patricia A. Reuter-Lorenz. We thank Thad Polk and Katherine Sledge Moore for their helpful comments on an earlier version of this manuscript.

doi:10.1016/j.neuroimage.2010.04.267 [Epub ahead of print].

Dale, A. M. (1999). Optimal experimental design for event-related fMRI. Hum. Brain Mapp. 8 , 109-114.

Daselaar, S., Rombouts, S., Veltman, D., Raaijmakers, J., and Jonker, C. (2003). Similar network activated by young and old adults during the acquisition of a motor sequence. Neurobiol. Aging 24, 1013-1019.

Dinstein, I., Gardner, J. L., Jazayeri, M. and Heeger, D. J. (2008). Executed and observed movements have different distributed representations in human aIPS. J. Neurosci. 28, 11231-11239.

Duverne, S., Motamedinia, S., and Rugg, M. (2009). The relationship between aging, performance, and the neural correlates of successful memory encoding. Cereb. Cortex 19, 733-744.

Friston, K. J., Holmes, A. P., Worsley, K. J., Poline, J. P., Frith, C. D., and Frackowiak, R. S. J. (1995). Statistical parametric maps in functional imaging: a general linear approach. Hum. Brain Mapp. 2, 189-210.

Haushofer, J., Livingstone, M., and Kanwisher, N. (2008). Multivariate 
patterns in object-selective cortex dissociate perceptual and physical shape similarity. PLoS Biol. 6, e187. doi: 10.1371/journal.pbio.0060187.

Haxby, J., Gobbini, I., Furey, M., Ishai, A., Schouten, J., and Pietrini, P. (2001). Distributed and overlapping representations of faces and objects in ventral temporal cortex. Science 293, 2425-2430.

Haynes, J. D., and Rees, G. (2006). Decoding mental states from brain activity in humans. Nat. Rev. Neurosci. 7, 523-534.

Hua, T., Li, X., He, L., Zhou, Y., Wang, Y., and Leventhal, A. G. (2006). Functional degradation of visual cortical cells in old cats. Neurobiol. Aging 27, 155-162.

Hutchinson, S., Kobayashi, M., Horkan, C. M., Pascual-Leone, A., Alexander, M. P., and Schlaug, G. (2002). Agerelated differences in movement representation. Neuroimage 17, 1720-1728.

Jonides, J., Schumacher, E., Smith, E., Lauber, E., Awh, E., Minoshima, S., and Koeppe, R. (1997). Verbal working memory load affects regional brain activation as measured by PET. J. Cogn. Neurosci. 9, 462-475.

Kriegeskorte, N., Goebel, R., and Bandettini, P. (2006). Informationbased functional brain mapping. Proc. Natl. Acad. Sci. U.S.A. 103, 3863-3868.

Leventhal, A. G., Wang, Y., Pu, M., Zhou, Y., and Ma, Y. (2003). GABA and its agonists improved visual cortical function in senescent monkeys. Science 300, 812-815.

Li, S., Lindenberger, U., and Sikström, S. (2001). Aging cognition: from neuromodulation to representation. Trends Cogn. Sci. (Regul. Ed.) 5, 479-486.

Li, S.-C., and Sikström, S. (2002). Integrative neurocomputational perspectives on cognitive aging, neuromodulation, and representation. Neurosci. Biobehav. Rev. 26, 795-808.

Lindenberger, U., and Baltes, P. B. (1994). Sensory functioning and intelligence in old age: a strong connection. Psychol. Aging 9, 339-355.

Mattay, V., Fera, F., Tessitore, A., Hariri, A., Berman, K., Das, S., MeyerLindenberg, A., Goldberg, T., Callicott, J., and Weinberger, D. (2006). Neurophysiological correlates of agerelated changes in working memory capacity. Neurosci. Lett. 392, 32-37.

Mattay, V. S., Fera, F., Tessitore, A., Hariri, A. R., Das, S., Callicott, J. H., and Weinberger, D. R. (2002). Neurophysiological correlates of agerelated changes in human motor function. Neurology 58, 630-635.

McEvoy, L. K., Pellouchoud, E., Smith, M. E., and Gevins, A. (2001). Neurophysiological signals of working memory in normal aging. $\operatorname{Cog} n$. Brain Res. 11, 363-376.

Miller, S., Celone, K., DePeau, K., Diamond, E., Dickerson, B., Rentz,D., Pihlajamäki, M., and Sperling, R. (2008). Age-related memory impairment associated with loss of parietal deactivation but preserved hippocampal activation. Proc. Natl. Acad. Sci. U.S.A. 105, 2181-2186.

Norman, K. A., Polyn, S. M., Detre, G. J., and Haxby, J. V. (2006). Beyond mindreading: multi-voxel pattern analysis of fMRI data. Trends Cogn. Sci. (Regul. Ed.) $10,424-430$.

Park, D. C., Polk, T. A., Park, R., Minear, M., Savage, A., and Smith, M.R. (2004). Aging reduces neural specialization in ventral visual cortex. Proc. Natl. Acad. Sci. U.S.A. 101, 13091-13095.

Park, D. C., and Reuter-Lorenz, P. (2009). The adaptive brain: aging and neurocognitive scaffolding. Annu. Rev. Psychol. 60, 173-196.

Park, J., Carp, J., Hebrank, A., Park, D., and Polk, T. (2010). Neural specificity pre- dicts fluid processing ability in older adults. J. Neurosci. 30, 9253-9259.

Peelen, M.V., Wiggett, A. J., and Downing, P. E. (2006). Patterns of fMRI activity dissociate overlapping functional brain areas that respond to biological motion. Neuron 49, 815-822.

Persson, J., Lustig, C., Nelson, J., and ReuterLorenz, P. (2007). Age differences in deactivation: a link to cognitive control? J. Cogn. Neurosci. 19, 1021-1032.

Postle, B. R., Zarahn, E., and D'Esposito, M. (2000). Using event-related fMRI to assess delay-period activity during performance of spatial and nonspatial working memory tasks. Brain Res. Brain Res. Protoc. 5, 57-66.

Rajah, N., and D'Esposito, M. (2005). Region-specific changes in prefrontal function with age: a review of PET and fMRI studies on working and episodic memory. Brain 128, 1964-1983.

Reuter-Lorenz, P., and Cappell, K. (2008). Neurocognitive aging and the compensation hypothesis. Curr. Dir. Psychol. Sci. 17, 177-182.

Reuter-Lorenz, P., and Park, D. (2010) Human neuroscience and the aging mind: a new look at old problems. $J$ Gerontol. 65, 405-415.

Reuter-Lorenz, P., Stanczak, L., and Miller A. (1999). Neural recruitment and cognitive aging: two hemispheres are better than one, especially as you age. Psychol. Sci. 10, 494-500.

Reuter-Lorenz, P. A., Jonides, J., Smith, E. E., Hartley, A., Miller, A., Marshuetz, C., and Koeppe, R. A. (2000). Age differences in the frontal lateralization of verbal and spatial working memory revealed by PET. J. Cogn. Neurosci. 12, 174-187.

Reuter-Lorenz, P.A., and Lustig, C. (2005) Brain aging: reorganizing discoveries about the aging mind. Curr. Opin. Neurobiol. 15, 245-251.

Rypma, B., and D'Esposito, M. (2000). Isolating the neural mechanisms of age-related changes in human working memory. Nat. Neurosci. 3, 509-515.

Rypma, B., Prabhakaran, V., Desmond, J.E., Glover, G. H., and Gabrieli, J.D. (1999). Load-dependent roles of frontal brain regions in the maintenance of working memory. Neuroimage 9, 216-226.

Schmolesky, M. T., Wang, Y., Pu, M., and Leventhal, A. G. (2000). Degradation of stimulus selectivity of visual cortical cells in senescent rhesus monkeys. Nat. Neurosci. 3, 384-390.

Schneider-Garces, N., Gordon, B., Brumback-Peltz, C., Shin, E., Lee, Y., Sutton, B., Maclin, E., Gratton, G., and Fabiani, M. (2010). Span, CRUNCH, and beyond: working memory capacity and the aging brain. J. Cogn. Neurosci. 22, 655-669.

Smith, E. E., Jonides, J., and Koeppe, R. A. (1996). Dissociating verbal and spatial working memory using PET. Cereb. Cortex 6, 11-20.

Conflict of Interest Statement: The authors declare that the research was conducted in the absence of any commercial or financial relationships that could be construed as a potential conflict of interest.

Received: 31 July 2010; accepted: 21 October 2010; published online: 22 November 2010.

Citation: Carp J, Gmeindl L and ReuterLorenz PA (2010) Age differences in the neural representation of working memory revealed by multi-voxel pattern analysis. Front. Hum. Neurosci. 4:217. doi: 10.3389/ fnhum.2010.00217

Copyright (c) 2010 Carp, Gmeindl and Reuter-Lorenz. This is an open-access article subject to an exclusive license agreement between the authors and the Frontiers Research Foundation, which permits unrestricted use, distribution, and reproduction in any medium, provided the original authors and source are credited. 